\title{
FATORES DIFICULTADORES NA GESTÃO DA QUALIDADE ASSISTENCIAL DE ENFERMAGEM EM UNIDADES DE TERAPIA INTENSIVA
}

\author{
Danielle P. O. Ribeiro*1, Maria A. Barbosa1, Marcos R. Fernandes¹, Ludimila C. S. Silva1, Maria C. \\ C. A. M. Queiroz ${ }^{2}$, Júlio C. S. Silva ${ }^{1}$, Marinésia A. Prado ${ }^{3}$ and Edinamar A. S. Silva ${ }^{1}$
}

${ }^{1}$ Programa de Pós-Graduação em Ciências da Saúde, Faculdade de Medicina, Universidade Federal de Goiás, Goiânia, Goiás, Brasil; ${ }^{5}$ Hospital das Clínicas da Universidade Federal, Goiânia, Goiás, Brasil; ${ }^{3}$ Departamento de Enfermagem, Faculdade de Enfermagem, Universidade Federal de Goiás, Goiânia, Goiás, Brasil

\section{ARTICLE INFO}

\section{Article History:}

Received $11^{\text {th }}$ March, 2021

Received in revised form

$03^{\text {rd }}$ April, 2021

Accepted $08^{\text {th }}$ May, 2021

Published online $30^{\text {th }}$ June, 2021

\section{Key Words:}

Diagrama de Ishikawa; Ferramenta de qualidade; Gestão de Enfermagem; Gestão da qualidade.

\section{*Corresponding author: Danielle P. O. Ribeiro}

\begin{abstract}
O objetivo foi conhecer os fatores dificultadores da gestão da qualidade por enfermeiros gestores nas Unidades de Terapia Intensiva. Estudo qualitativo, em uma instituição de Saúde Pública do Brasil, com a participação de 18 enfermeiros. Entrevistas, com questões abertas acerca das variáveis independentes: caracterização dos profissionais (sexo, idade, escolaridade, local de atuação, tempo de atuação profissional) e desfecho: fatores dificultadores acerca da gestão da qualidade assistencial, antes, durante e depois da intervenção educativa. Dados organizados com o auxílio do software ATLAS $t i$ e a análise de conteúdo de Bardin, resultando em três Diagramas de Ishikawa: pré-intervenção, intervenção e pós-intervenção, emergindo nas causas raiz: déficit dimensionamento (pré-intervenção e intervenção), insubordinação e relacionamento interpessoal (pré e pós-intervenção), alto índice de absenteísmo (intervenção e pós-intervenção), déficit profissionais com capacitação técnica (intervenção e pós-intervenção). A utilização do Diagrama de Ishikawa nas etapas do estudo, auxiliou na identificação das causas-raiz apontadas pelos sujeitos da pesquisa identificando os pontos críticos relacionados aos fatores dificultadores da gestão da qualidade assistencial. Além disso, destaca-se a necessidade do preparo do gestor de enfermagem, em conceitos e técnicas relacionados a gestão da qualidade assistencial, como estratégias, planos de ação e metas que deverão ser elaboradas, entendidas e praticadas.
\end{abstract}

Copyright (C) 2021, Danielle P. O. Ribeiro et al. This is an open access article distributed under the Creative Commons Attribution License, which permits unrestricted use, distribution, and reproduction in any medium, provided the original work is properly cited.

Citation: Danielle P. O. Ribeiro, Maria A. Barbosa, Marcos R. Fernandes, Ludimila C. S. Silva, Maria C. C. A. M. Queiroz, Júlio C. S. Silva, Marinésia A. Prado and Edinamar A. S. Silva. 2021. "Fatores dificultadores na gestão da qualidade assistencial de enfermagem em unidades de terapia intensiva", International Journal of Development Research, 11, (06), 47977-47980.

\section{INTRODUCTION}

Gestão da Qualidade é uma filosofia baseada na satisfação dos clientes internos e externos envolvidos na empresa, ou seja, é um meio para atingir os objetivos e resultados desejados, e como tal, faz uso de um conjunto de técnicas e ferramentas integradas ao modelo para analisar fatos e auxiliar na tomada de decisão (KRAU, 2019). Esses instrumentos utilizados visam obter um grau de eficiência em uma determinada atividade ou processo (WHITING et al., 2017). A gestão, a qualidade e a saúde associadas podem melhorar e revolucionar sistemas, a fim de promover mudanças, mobilizar caminhos, bem como convocar protagonistas e detectar paisagem interativa de indivíduos. É importante considerar a inter-relação destas várias linhas e a preocupação com o desenvolvimento de métodos e condutas compartilhados num permanente aprender a aprender (MELO et al., 2014). Os serviços de Saúde emergenciais, tem como objetivos a completa satisfação e recuperação dos pacientes, resultando na eficiência operacional da Instituição. Assim é de suma importância que a ferramenta escolhida faça parte do arsenal de apoio à função gerencial do enfermeiro, sendo exequível por toda equipe, pois ele tem sido cada vez mais o definidor da qualidade no cuidado prestado (OLIVEIRA et al., 2017). O Diagrama de Ishikawa, simplifica processos considerados complexos dividindo-os em algo mais simples e, mais controláveis. Essa ferramenta permite também, estruturar qualquer sistema que necessite de resposta de forma gráfica e sintética, para uma melhor visualização e consequente compreensão do conteúdo (LILIANA, 2016).

A implantação e manutenção de uma eficiente gestão da qualidade assistencial, é um grande desafio, uma vez que é necessário o envolvimento de pessoas, processos, qualificação e tecnologias, além da compreensão de como se efetiva a qualidade e a percepção dos diferentes atores sociais que participam nesse processo, nos vários níveis de ação em saúde, resultando em um desafio constante a ser realizado pelo gestor. Frente ao exposto, o objetivo do presente estudo foi identificar os fatores dificultadores relacionados a gestão da qualidade assistencial apontados pelos gestores de enfermagem das Unidades de Terapia Intensiva. 


\section{MATERIAIS E MÉTODOS}

Trata-se de estudo qualitativo inserido no projeto âncora intitulado: Processo de Auditoria em instituição de saúde de Goiás, realizado em um hospital público de grande porte da região central do Brasil, especializado no atendimento materno-infantil, no período entre outubro de 2018 a agosto de 2019. A amostragem do estudo foi não probabilística. Os gestores de enfermagem da Unidade de Terapia Intensiva neonatal, pediátrica, e materna e Semi-intensiva neonatal que tivessem vínculo empregatício com a instituição foram incluídos. Aqueles profissionais que se encontravam de licença médica, maternidade, férias ou foram transferidos, em uma das duas fases da coleta de dados, foram excluídos do estudo. Estabelecido contato prévio com a gerente de Enfermagem da Instituição e com as gestoras participantes para definição do local das entrevistas, com posterior envio de convite individual e nominal a cada uma, contendo data, horário e local do agendamento. Realizadas na sala de reuniões da unidade de terapia intensiva neonatal, de modo individual e sem interrupções, proporcionando ampla exploração sobre o assunto, mediante as informações fornecidas por cada participante. As entrevistas foram realizadas apenas pela pesquisadora do estudo e iniciadas com a explicação dos objetivos da pesquisa, entrega e leitura em conjunto. $\mathrm{Na}$ sequência realizamos as perguntas com a gravação do material no intuito de manter a fidedignidade dos dados durante as transcrições das respostas. As interlocuções ocorreram antes e depois da intervenção, o que denominamos pré-intervenção (outubro e novembro de 2018) e pós-intervenção (julho e agosto de 2019).

A intervenção educativa ocorreu após a análise das percepções dos en-fermeiros sobre gestão da qualidade assistencial em saúde na préintervenção, subsidiando na determinação dos eixos temáticos e norteando a elaboração da ação educativa, desenvolvida acerca de aspectos científicos, sobre mapa conceitual (definição, objetivos e tipos), Brainstorming, gestão da qualidade da assistência e as ferramentas da qualidade. Em seguida, os participantes realizaram a construção de um mapa conceitual sobre os fatores dificultadores na gestão da qualidade assistencial, identificados na própria instituição e na sequência o Brainstorming agrupando e condensando ideias sobre estratégias para solucionar os problemas evidenciados,fornecendo dados para a construção do diagrama de Ishikawa. Essa atividade foi desenvolvida em um único módulo, na própria instituição, nos meses de fevereiro e março de 2019, com vagas disponibilizadas de acordo com o turno de trabalho de cada profissional. $\mathrm{O}$ instrumento utilizado para coleta de dados consistiu em sete perguntas abertas, acerca da percepção, aspectos positivos e negativos e fatores dificultadores em relação a gestão da qualidade asisstencial, e foi utilizado para avaliar a gestão da qualidade assistencial (RABENSCHLAG, 2015). As variáveis independentes foram: sexo (masculino/feminino), idade (em anos vividos), local de atuação (UTI neonatal/ pediátrica/ materna/Semi-intensiva neonatal), escolaridade (em anos formais), especialização (sim/não) e tempo de atuação profissional (em anos). $\mathrm{O}$ desfecho do estudo foram os fatores dificultadores acerca da gestão da qualidade assistencial coletados pelo instrumento descrito acima. Os dados foram submetidos à análise de conteúdo de Bardin (BARDIN, 2011), em seguida utilizou-se o ATLAS.ti, um Software de Tecnologia da Informação para a organização dos dados advindos dos fragmentos de textos significativos dos relatos dos profissionais (OLIVEIRA et al., 2017), na pré e pós intervenção, e na intervenção, utilizou-se o mapa conceitual, para a organizaçãodas palavras-chave, com posterior construção dos diagramas de Ishikawa. O protocolo de pesquisa foi devidamente aprovado pelo Comitê de Ética em Pesquisa do Hospital Materno Infantil, sob o número CEPES/HMI/GO 1.447.390.

\section{RESULTADOS}

Entre os 30 enfermeiros que atuam nas Unidades de Terapia Intensiva (materna, pediátrica e neonatal) e Unidade de Terapia Semi-Intensiva neonatal, 28 (93\%) participaram da entrevista na pré-intervenção, e
18 (64\%) na pós intervenção. Os 10 (36\%) não participantes da préintervenção, foram excluídos por motivos variados como desligamento, transferência, licença médica e licença maternidade, atendendo aos critérios pré-estabelecidos. Dessa forma a amostragem final do estudo foi de 18 enfermeiros elegíveis por participarem das duas fases. Os dados sociodemográficos e de caracterização profissional estão dispostos na Tabela 1 .

Tabela 1. Dados sociodemográficos e profissionais da amostra do estudo $(n=18)$

\begin{tabular}{|c|c|c|c|c|}
\hline Variáveis & $\mathrm{M}$ & (DP) & $\mathrm{N}$ & $\%$ \\
\hline Idade & 41 & 8 & 18 & $100 \%$ \\
\hline \multirow[t]{2}{*}{ Sexo } & $\mathrm{F}$ & & 18 & $100 \%$ \\
\hline & M & & 0 & $0 \%$ \\
\hline \multirow{4}{*}{ Local de atuação } & UT & tal & 3 & $17 \%$ \\
\hline & UT & rica & 3 & $17 \%$ \\
\hline & UT & & 4 & $22 \%$ \\
\hline & UT & Intensiva & 8 & $44 \%$ \\
\hline Escolaridade (em anos & De & anos & 15 & $83 \%$ \\
\hline formais) & $\mathrm{Ma}$ & anos & 3 & $17 \%$ \\
\hline \multirow[t]{2}{*}{ Especialização } & Pos & & 18 & $100 \%$ \\
\hline & Nãc & & 0 & $0 \%$ \\
\hline Tempo de atuação & De & anos & 16 & $89 \%$ \\
\hline Profissional (em anos) & $\mathrm{Ma}$ & anos & 2 & $11 \%$ \\
\hline
\end{tabular}

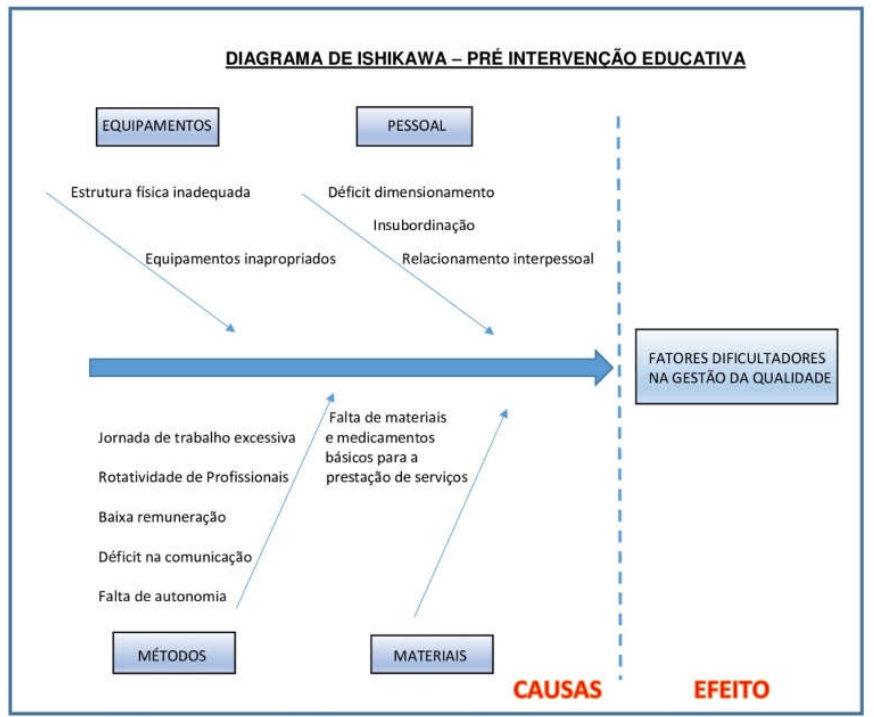

Figura 1. Diagrama de Ishikawa Pré-Intervenção Educativa

Diante das respostas às questões objetivas, foi solicitado às enfermeiras que discorressem sobre os fatores dificultadores em relação a gestão da qualidade.

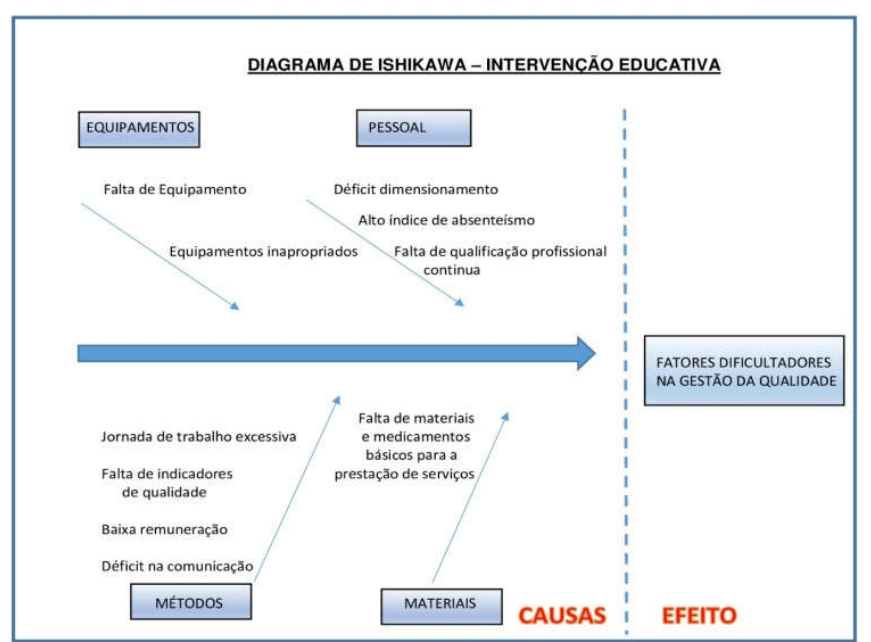

Figura 2. Diagrama de Ishikawa Intervenção Educativa 
Considerando os fatores elencados pelas gestoras de enfermagem durante a entrevista realizada antes da ação de intervenção educativa, as principais causas-raiz do problema (subgrupo) foram organizadas na Figura 1. As causas-raiz que emergiram na pré-intervenção acerca das 4 categorias foram: déficit de dimensionamento, insubordinação, relacionamento interpessoal, (pessoal); estrutura física inadequada e equipamentos inapropriados, (equipamentos); falta de materiais e medicamentos básicos para a prestação de serviços, (materiais); jornada de trabalho excessiva, rotatividade de profissionais, baixa remuneração, déficit na comunicação e falta de autonomia, (método). $\mathrm{Na}$ intervenção educativa, seguindo essas categorias, surgiram novas causas-raiz, como, alto índice de absenteísmo, poucas capacitações técnicas, falta de equipamentos e falta de indicadores de qualidade (Figura 2). Na pós-intervenção, os gestores de enfermagem apontaram algumas causas-raiz citadas na pré-intervenção e na intervenção educativa, com predominância de causas elencadas na intervenção, que deram origem ao diagrama de Ishikawa PósIntervenção Educativa, conforme demonstrado na Figura 3.

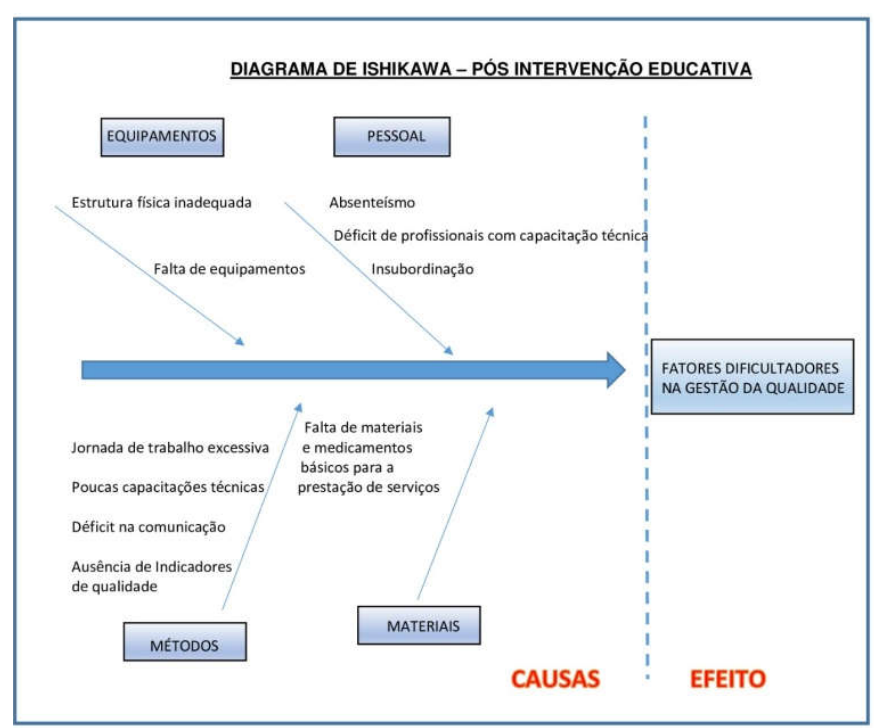

Figura 3. Diagrama de Ishikawa Pós-Intervenção Educativa

\section{DISCUSSÃO}

Os fatores dificultadores encontrados pelos gestores de enfermagem nas fases pré/ intervenção educativa/ pós, foram: déficit dimensionamento, insubordinação, relacionamento interpessoal, alto índice de absenteísmo, déficit profissionais com capacitação, estrutura física inadequada, equipamentos inadequados, falta de equipamentos, falta de materiais e medicamentos básicos para a prestação de serviços, jornada de trabalho excessiva, baixa remuneração, alta rotatividade de profissionais, déficit de comunicação, falta de autonomia, poucas capacitações técnicas ofertadas pela Instituição e falta de indicadores de qualidade. De acordo com as entrevistadas o déficit no dimensionamento foi apontado como uma das causas-raiz do problema, mesmo com o respaldo da Resolução Cofen 543/2017 (COFEN, 2019)que prevê o dimensionamento do quadro de profissionais de enfermagem baseado em características relativas ao serviço de saúde. Um dimensionamento inadequado, pode resultar em impactos irreversíveis, considerando que a assistência aos pacientes, leva à sobrecarga de trabalho da equipe de enfermagem. Sendo assim, o número escasso de profissionais também está, diretamente, relacionado à segurança do paciente (NUNES; TOMA, 2013). Os diferentes regimes trabalhistas, que são mesclados entre estatutários e celetistas, foram relatados pelas gestoras de enfermagem como um fator considerável que resulta na insubordinação a determinadas atividades propostas, uma vez que alguns estatutários são geridos por celetistas, resultando em conflitos interpessoais9. Esses conflitos podem gerar insubordinações, aumento do absenteísmo, além de outros problemas responsáveis pelo desequilíbrio das relações, colaborando para o desencadeamento de transtornos físicos e psíquicos nos trabalhadores, afetando diretamente na gestão da qualidade assistencial (CALHA et al., 2018; FERRARO, 2016).

A complexidade das atividades de enfermagem na Unidade de Terapia Intensiva (UTI), e a dependência de um conjunto infindável de necessidades para o desdobramento do cuidado, influenciam na performance dos profissionais de enfermagem. A condição complexa dos pacientes e a aplicação de variadas tecnologias, demandam da enfermagem conhecimentos diversos, potencializando métodos efetivos de trabalho e cuidado. Sendo assim a qualidade dos serviços de saúde e do cuidado em saúde dependem de uma conexão complexa do processo de trabalho da equipe multidisciplinar incluindo as condições de infraestrutura, dos serviços e do sistema de saúde, tendo a gestão um papel essencial nesta articulação (MOHAMMAD, 2018). No ambiente hospitalar, os estoques de insumo em saúde desempenham um papel de grande importância, viabilizando a assistência, considerando o desconhecimento e a variabilidade das necessidades dos pacientes, e que por esse motivo, devem ser geridos de forma eficiente. Entretanto, a dificuldade em organizar a previsão dos recursos materiais e em definir a relação entre oferta de serviços e demandas de saúde resulta na falta de qualidade da assistência à saúde (RAMOS, 2018).

A baixa remuneração e a falta de reconhecimento da profissão foram citados como consequências da desvalorização da força de trabalho do enfermeiro sendo fatores potencial geradores da jornada de trabalho excessiva. Assim, a ausência ou deficiência na comunicação são causas importantes acerca do clima desfavorável entre os membros da equipe com consequente alta rotatividade, fatores que interferem, significativamente na qualidade do cuidado prestado (MARTINS et al., 2019; YOO et al., 2016). A falta de autonomia, o déficit de conhecimento na área de atuação, bem como a exaustão de trabalho são obstáculos existentes no processo de trabalho do profissional enfermeiro, apontados como causas-raiz em diferentes fases do estudo (OSHODI et al., 2019). As gestoras de enfermagem reconheceram a importância da capacitação em saúde, como fonte contínua de fortalecimento e qualificação das suas ações contribuindo nos processos de atualização e auxiliando nas atividades que facilitam o aprendizado, troca de conhecimentos, saberes e reflexão dos processos de trabalho. Além disso, elas ressaltaram que além de serem pouco ofertadas pela instituição, fatores como sobrecarga de trabalho, déficit de dimensionamento, cargas horárias exaustivas e falta de recursos materiais dificultam a participação desses profissionais nas atividades de educação em saúde (MERSIN et al., 2018). A falta de indicadores emergiu durante a intervenção educativa, bem como na segunda fase (pós-intervenção), denotando um amadurecimento dos gestores de enfermagem acerca do processo de gestão da qualidade assistencial, uma vez que para medi-la se faz necessário o desenvolvimento de uma metodologia que possa auxiliar esses gestores na avaliação, análise e interpretação dos processos operacionalizados em suas atividades de trabalho (BAÓ et al., 2019). Apontamos como limitações deste estudo, a amostra não probabilística, a não abrangência de todos os gestores de enfermagem, além do fato do estudo ter ocorrido em uma única instituição de saúde, limitando a capacidade de generalização do mesmo. Assim, mais estudos são necessários para avaliar os fatores dificultadores apontados pelos enfermeiros gestores acerca da gestão da qualidade assistencial, estimando a reprodutibilidade do estudo em questão, viabilizando sua complementação com metodologias distintas e possibilitando agregar novas estratégias para maior integração do tema.

\section{CONCLUSÃO}

Destacamos a importância do conhecimento do enfermeiro gestor acerca das relações de causa e efeito que interagem entre si, para o entendimento dos pontos críticos e análise de todos os fatores que envolvem a execução do processo, permitindo a organização das informações e identificação das possíveis causas relacionadas aos problemas. Esses foram os fatores dificultadores encontrados no estudo: déficit de dimensionamento, insubordinação, relacionamento interpessoal, alto índice de absenteísmo, déficit de profissionais com 
capacitação técnica, estrutura física e equipamentos inadequados, falta de equipamentos e falta de materiais e medicamentos básicos para a prestação de serviços em saúde. Fatores que até então, não estavam bem definidos entre os gestores de enfermagem. É possível evidenciar cada uma das causas e a que elas estão relacionadas para influenciar na continuação do processo utilizando demais ferramentas da qualidade no processo de melhorias e na efetivação de ações corretivas eficazes.

\section{AGRADECIMENTOS}

Os autores agradecem a todos os participantes do Programa de PósGraduação em Ciências da Saúde da Faculdade de Medicina da Universidade Federal de Goiás e aos Sujeitos da Pesquisa.

\section{REFERÊNCIAS}

Baó, A. C. P., Amestoy, S. C., Moura, G. M. S. S., Trindade, L. L. Indicadores de qualidade: ferramentas para o gerenciamento de boas práticas em saúde. Rev Bras Enferm. 2019;72(2):377-84.

Bardin, L. (2011). Análise de conteúdo. Vol. I, Edições 70, São Paulo, Brasil.

Calha, A., Ferreira, M., Alminhas, S., Pequito, T. Interpersonal conflict management strategies in nursing teams. Revista de enfermeria. 2018 [ citado 22 nov. 2020]. Disponível em: https://www.researchgate.net/publication/329454478_Interperso nal_conflict_management_strategies_in_nursing_teams.

Conselho Federal de Enfermagem - Cofen. Resolução 543/2017 [citado 18 jun. 2019]. Disponível em: http://www.cofen.gov.br/resolucao-cofen-5432017 51440.html.

Ferraro, C. Incidencia de ausentismo laboral y factores determinates em el personal de enfermeria del hospital zonal especializado em oncologia "Luciano Fortabat" de Olavarria [dissertação] [internet]. Rosário: Universidad Nacional de Rosario; 2016. [citado 20 nov. 2019]. Disponível em: http://capacitasalud.com/ biblioteca/wp-content/uploads/2016/07/TESIS-FINAL.pdf

Krau, S. D.Quality Improvement: Evolution or Revolution? Nurs Clin N Am. 2019;54.

Liliana, L. A new model of Ishikawa diagram for quality assessment. IOP Conference Series Materials. Science and Engineering. 2016; 161(1):012099.
Martins, M. S., Matos, E., Salum, N. C. Rotatividade Dos Trabalhadores De Enfermagem Em Uma Unidade De Emergência Adulto. Rev.Texto contexto - enferm. 2019; 28.

Melo, W. O. S., Maia, A. E. S., Barracho, A. A., Ribeiro, B. P., Correa, C. J. A. G., Correa, I. G, et al. Gestão Da Qualidade Na Saúde. Rev. UNINGÁ Review. 2014 [citado 01 abr. 2019];18(1):24-28. Disponível em: \&lt;https://www. mastereditora.com.br/periodico/20140331_190319.pdf\&gt

Mersin, S., İpçioğlu, I., Koca, G. Analysis of the Workload of Nurses. Health Care Academician Journal. 2018.

Mohammad, E. E. H. Intensive Care Unit Nurses' Performance Regarding Caring Patients With Head Injury: An Educational Intervention. International Journal of Studies in Nursing.,2018; $3(3)$.

Nunes, B. K., Toma, E. Dimensionamento de pessoal de enfermagem de uma unidade neonatal: utilização do Nursing Activities Score. Rev. Latino Americana de Enfermagem. 2013.

Oliveira, S. A., Almeida, M. L., Santos, M. F., Zilly, A., Peres, A. M., Rocha, F. L. R., et al. Ferramentas gerenciais na prática de enfermeiros da atenção básica em saúde. Rev. Adm. Saúde. 2017; 17(69).

Oshodi, T. O., Bruneau, B., Crockett, R., Kinchington, F., Nayar, S., West, E. Registered nurses' perceptions and experiences of autonomy: a descriptive phenomenological study. BMC Nursing. 2019; 18(51).

Rabenschlag, L. A., Lima, S. B. S., Eberhardt, T. D., Kessler, M., Soares, R. S. A., Camponogara, S. Gestão da Qualidade na Assistência de Enfermagem em Unidade de Clínica cirúrgica. 2015. Rev. Enferm. UFSM. 2015; 5(2): 235-246.

Ramos, L. C. F., Spiegel, T., Assad, D. B. N. Gestão de materiais hospitalares: uma proposta de melhoria de processos aplicada em hospital universitário. Rev. Adm. Saúde. 2018; 18(70).

Whiting, P., Wolff, R., Mallett, S., Simera, I., Savović, J. A proposed framework for developing quality assessment tools. Systematic Reviews. 2017; 6:204.

Yoo, B. K, Kim, M., Lin, T. C., Sasaki, T., Ward, D., Spetz, J. The effect of prior healthcare employment on the wages of registered nurses. BMC Health Services Research. 2016; 16:412. 